\title{
Variants of Human Chorionic Gonadotropin from Pregnant Women and Tumor Patients recognized by Monoclonal Antibodies
}

\author{
PETER BERGER, SIEGFRIED SCHWARZ, GERALD SPÖTTL, GEORG WICK, AND \\ KLAUS MANN \\ Institute for Biomedical Aging Research of the Austrian Academy of Sciences (P.B., G.W.), Innsbruck, \\ Austria; Institute for General and Experimental Pathology (S.S., G.W.), University of Innsbruck, Austria; \\ and Department of Internal Medicine II (G.S., K.M.), Klinikum Grosshadern, University of Munich, Federal \\ Republic of Germany
}

\begin{abstract}
In biological fluids, hCG and its free $\alpha-(\mathrm{hCG} \alpha)$ and $\beta$-subunits (hCG $\beta$ ), occur in multiple forms. These various forms differ at the molecular level primarily in glycosylation, but also differ in protein backbone modifications corresponding to the urinary low molecular weight fragment of the hCG $\beta$-subunit ( $\beta$-core fragment). This microh eterogeneous nature can be demonstrated by isoelectric focusing in which variants are separated into bands with different isoelectric points (pI). To determine whether such isoelectric variants differ in antigenicity and consequently might escape immunoassay detection due to overspecificity of monoclonal antibodies (MCA), urinary pregnancy hCG (NIH, CR123) and tumor hCG preparations, such as a tumorspecific acidic variant of hCG (hCGav) and the $\mathrm{hCG} \beta$-core fragment were separated by isoelectric focusing in the absence or presence of 8 $\mathrm{M}$ urea, or by sodium docedyl sulfate-polyacrylamide gel electrophoresis
\end{abstract}

and enzymatically immunostained using an MCA panel directed against 17 different hCG epitopes. MCA against 14 different epitopes accessible on holo-hCG recognized all pI variants of pregnancy holohCG or tumor-derived hCGav, as was true for the three MCA recognizing epitopes hidden on holo-hCG but accessible on the free subunits after hCG dissociation by urea.

We conclude that each individual pI-isoform of holo-hCG and its free subunits expresses the entire set of epitopes recognized by our MCA panel. The carbohydrate moieties that form a biochemical basis for hCG heterogeneity seem to be neither of major antigenic relevance, nor are they structurally related to any particular epitope. Thus, various glycosylation forms of hCG, hCG $\alpha, \mathrm{hCG} \beta$, and hCG $\beta$-core in normal as well as in pathological samples should safely be detectable and measureable by immunoassays employing MCA with appropriate subunit specificity. (J Clin Endocrinol Metab 77: 347-351, 1993)
A S ANY other glycoprotein hormone, hCG is composed of $\alpha-(\mathrm{hCG} \alpha)$ and $\beta$-subunits (hCG $\beta$ ) noncovalently linked after postranslational modifications (reviewed in 1 , 2). Physiologically, hCG is mainly a secretory product of the placental syncytiotrophoblast. For trophoblastic diseases, such as choriocarcinoma, hydatidiform mole, and nonseminomatous testicular cancer, hCG is an established tumor marker $(1,3)$. Apart from the holo-hormone, tumors secrete variable quantities of free hCG $\alpha$ and/or hCG $\beta$ (4-8). Glycoprotein hormones, such as hCG, have been shown to occur in heterogeneous forms rather than as homogeneous molecular entities. This microheterogeneity is generally understood to result from posttranslational modifications. Glycosylation variants with varying degrees of negatively charged terminal sialic acids (9-11) consequently differ in their net charge (12), and thus in their isoelectric points ( $\mathrm{pI}$ ).

We have developed a panel of monoclonal antibodies (MCA) against hCG (13), hFSH (14), bLH (15), and against the free subunits of hCG (4) that allowed us to determine the number and topographical arrangement of epitopes on hCG $(4,16)$. On this basis, we were able to construct epitopeselective immunoassays for the detection of holo-hCG and

Received May 1, 1991

Address correspondence and requests for reprints to: Dr. P. Berger, Institute for Biomedical Aging Research of the Austrian Academy of Sciences, Fritz-Pregl-Strasse 3/IV, A-6020 Innsbruck, Austria. its free subunits, respectively $(4,8)$. Further, we developed a sensitive and effective method to analyze the electrical charge heterogeneity of hCG by isoelectrofocusing (IEF) and immunoblotting. A highly acidic variant of hCG (hCGav) was detected in any patient presenting with trophoblastic tumor. hCGav is nearly absent during normal pregnancy and therefore apparently indicative for tumor-derived hCG $(3,17)$.

Diagnostic immunoassays for hCG and its free subunits require that the antibodies employed are able to recognize all variants with similar affinity, or at least do not exclude one or several major molecular forms. Whereas overspecificity is no problem with polyclonal antisera which, a priori, have been raised against microheterogenous preparations of hCG or hCG $\beta$ it may become relevant when MCA are used (8).

Since the above-mentioned hCGav can comprise up to $45 \%$ of the total hCG content in serum (17), and, since hCG $\beta$-core is one of the major urinary hCG-derived components $(18,19)$, the question of their antigenic properties arose. The contribution of normal pregnancy-derived or oversized tumor hCG carbohydrate moieties to immunological epitopes could have major impact on the reliable detection and measurement of tumor-derived hCG variants by MCA based immunoassays. This issue may be particularly crucial if proportions of variants differ at certain stages of tumorigenesis or tumor recurrence. We therefore addressed 
the question of whether variants of normal pregnancy hCG, abnormal tumor hCG, and free hCG subunits can be recognized by our MCA and thus expose all previously described epitopes.

\section{Materials and Methods}

\section{$M C A$}

MCA against hCG, free $h C G \alpha$, free $h C G \beta, h F S H$, and bLH were characterized as to affinity and subunit-specificity by one-site double antibody direct binding and competitive RIA and enzyme-linked immunosorbent assay (ELISA) $(13,4,14,15)$. Epitope specificity was assessed by two-site immunoassays $(4,14,16)$. Seventeen different epitopes can be distinguished on hCG and its subunits. The 14 epitopes which are accessible on holo-hCG are termed according to their subunit localization: $\alpha 1$ to $\alpha 5, \beta 1$ to $\beta 5$, and $c 1$ to $c 4$, the latter referring to "conformational" (c) $\alpha \beta$ epitopes exclusively exposed by holo-hCG. Three epitopes, $\alpha 6, \beta 6$ and $\beta 7$, are present only on the nonassociated subunits (hidden epitopes). The 17 epitopes are recognized by the following 17 MCA: INN(sbruck)-hFSH-73 (epitope $\alpha 1$ ), INN-hFSH-98 $(\alpha 2)$, INN-hCG-15 $(\alpha 3)$, INN-hFSH-132 $(\alpha 4)$, INN-hFSH-158 $(\alpha 5)$, INN-hCG-72 $(\alpha 6)$, INN-hCG-2 $(\beta 1)$, INN-hCG-22 ( $\beta 2)$, INN-bLH-1 $(\beta 3)$, INN-hCG-24 ( $\beta 4)$, INN-hCG-58 ( $\beta 5)$, INN-hCG-64 ( $\beta 6)$, INNhCG-68 ( $\beta 7)$, INN-hCG-10 (c1), INN-hCG-40 (c2), INN-hCG-45 (c3), and INN-hCG-26 (c4).

\section{Purification of the hCGav and of the hCG $\beta$-core fragment}

hCGav was purified, as described previously (17), from the urine of patient H.K., who had metastatic nonseminomatous testicular cancer. Urine from patient C.F., who also suffered from nonseminomatous testicular cancer, was concentrated in an ultrafiltration cell (Amicon Corporation, Lexington, Mass.) with a molecular filter YM-5 (5 kD cut off) and thereafter lyophilized. An aliquot of $300 \mu \mathrm{g}$ was dissolved in 3 $\mathrm{mL}$ distilled water, applied to a $2.5 \times 100 \mathrm{~cm}$ Ultrogel AcA 44 column (Serva, Heidelberg, FRG) and eluted at $4 \mathrm{C}$ with $30 \mathrm{~mL} / \mathrm{h} 0.01 \mathrm{M}$ Tris$\mathrm{HCl} / 0.15 \mathrm{M} \mathrm{NaCl}$ ( $\mathrm{pH} 8.0$ ). Marker proteins were run for mol wt estimation. Fractions of $9 \mathrm{~mL}$ were measured simultaneously by two immunoradiometric assays specific for holo-hCG and the free hCG $\beta$ subunit, respectively (7). Those fractions devoid of holo-hCG but containing low mol wt fragments with immunoreactivity for hCG $\beta$ (hCG $\beta$ core fraction) were pooled, concentrated, and rechromatographed as described above. Enriched hCG $\beta$-core was extensively dialyzed against ammonium bicarbonate $(0.01 \mathrm{~m})$ and lyophilized. Its apparent $\mathrm{mol} \mathrm{wt}$ was 12,000 . This material will be referred to as less than $12 \mathrm{kDa}$ fraction, in contrast to the greater than $12 \mathrm{kDa}$ fraction which contained holohCG and free hCG $\beta$.

\section{Polyacrylamide gel IEF}

Preparative IEF in granulated gels (pH gradient 3-6; ampholytes, Serva, Heidelberg, FRG) was performed according to manufacturer's instructions (LKB, Bromma, Sweden). Analytical IEF ( $\mathrm{pH}$ gradient 3-10) of purified hCG preparations was performed on polyacrylamide gels as described previously (17). Samples from patients or standard preparations $(100 \mu \mathrm{l} ; 1-10 \mathrm{mg}$ protein $/ \mathrm{ml})$ were applied to polyacrylamide gels (size: $160 \times 60 \times 0.5 \mathrm{~mm}$ ) with filter paper strips (Whatman no. $1 ; 135$ $\times 3 \mathrm{~mm}$ ) and focused: $\mathrm{T}=7.7 \%, \mathrm{C}=3 \%$; $\mathrm{pH}$ gradient $3-10$; water cooling; constant power was set at $15 \mathrm{~W}$ for $90 \mathrm{~min}$.

\section{Western blot analysis}

Proteins resolved by IEF or sodium dodecyl sulfate-polyacrylamide gel electrophoresis (SDS-PAGE) were electrophoretically transferred to nitrocellulose sheets (Biotec-Fischer, Reiskirchen, FRG) at $200 \mathrm{~mA}$ for 1 $h$. Lanes containing the mol wt standards were then removed and stained with india ink. Sample lanes were treated for $5 \mathrm{~min}$ with sample buffer $(0.05 \mathrm{M}$ Tris $-\mathrm{HCl} / 0.15 \mathrm{M} \mathrm{NaCl} / 0.5 \%$ Tween $20 / \mathrm{pH} 10.3)$ and then incubated in an incubation manifold for $1 \mathrm{~h}$ at $20 \mathrm{C}$ with either of the $17 \mathrm{MCA}$ (ascites diluted 1:200 in sample buffer) against the 17 different hCG epitopes. After extensive washing of the sheets with sample buffer without Tween 20 , pI bands were detected by an alkaline phosphataseconjugated antimouse immunoglobulin $\mathrm{G} \mathrm{F(ab)})_{2}$ antibody (Zymed, San Francisco, CA; 1:500 in sample buffer, $30 \mathrm{~min}$ ). Removal of excess conjugate by thorough washing was followed by addition of the substrate (5-bromo-4-chloro-3-indolyl-phosphate/nitro blue tetrazolium).

\section{Results}

\section{Pregnancy $h C G$}

Highly purified urinary standard preparations of pregnancy holo-hCG and its isolated subunits were run on SDSgels, transferred to nitrocellulose sheets, and stained in parallel with MCA. In these Western blots holo-hCG (NIH, CR123) appeared as a single band between $47 \mathrm{kDa}$ and 63 $\mathrm{kDa}$, free hCG $\beta$ (NIH, CR123B) between $33 \mathrm{kDa}$ and $45 \mathrm{kDa}$ and free hCG $\alpha$ (NIH, CR123A) between $20 \mathrm{kDa}$ and $28 \mathrm{kDa}$ (Fig. 1). These relative size differences on SDS-PAGE correspond to the different mol wt as determined by gel chromatography and other methods (2).

Since all lanes within one blot contained equal amounts of antigen, differences in staining intensity between MCA reflected differences in MCA affinity. Staining strength differences between two antigen preparations (holo-hCG, free subunit) of a given MCA is likely due to conformational

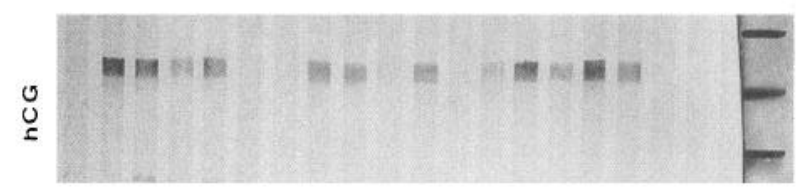
MW $67 \mathrm{kD}$ $43 \mathrm{kD}$ $30 \mathrm{kD}$ $\alpha_{1} \alpha_{2} \alpha_{3} \alpha_{4} \alpha_{5} \alpha_{6} \quad$ c1 c2 c3 c4 $\quad \beta_{1} \beta_{2} \beta_{3} \beta_{4} \beta_{5} \beta 6 \beta 7$

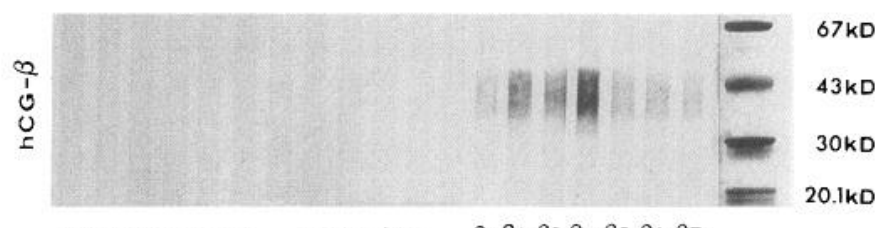
$\alpha_{1} \alpha_{2} \alpha_{3} \alpha_{4} \alpha_{5} \alpha_{6} \quad$ cl c2 c3 c4 $\quad \beta_{1} \beta_{2} \beta_{3} \beta_{4} \beta 5 \beta_{6} \beta 7$

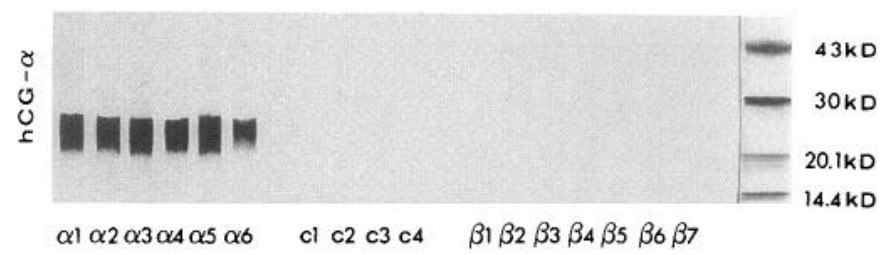

FIG. 1. Analysis of highly purified standard pregnancy hCG (NIH, CR123), hCG $\alpha$ (NIH, CR123A), and hCG $\beta$ (NIH, CR123B) by SDS. PAGE and Western blotting. Immunostaining with 17 different MCA against 17 distinct epitopes of hCG and its free subunits designated $\alpha 1$ to $\beta 7$. Development by an antimouse immunoglobulin G-alkaline phosphatase conjugate. The mol wt markers (to the right) phosphorylase $b$ $(94 \mathrm{k})$, BSA $(67 \mathrm{k})$, ovalbumin $(43 \mathrm{k})$, carbonic anhydrase $(30 \mathrm{k})$, soybean trypsin inhibitor $(20.1 \mathrm{k})$ and $\alpha$-lactalbumin $(14.4 \mathrm{k})$ were stained with India ink. Holo-hCG (upper panel), which is detected by MCA against public epitopes ( $\alpha 1$ to $\alpha 5, \beta 1$ to $\beta 5, \mathrm{c} 1$ to $\mathrm{c} 4$ ) appears as a broad single band with an apparent mol wt between $47 \mathrm{k}$ and $63 \mathrm{k}$. Free hCG $\beta$ subunit (mol wt: $33 \mathrm{k}-45 \mathrm{k}$ ) stained exclusively with $\beta$ MCA including the MCA directed against free hCG $\beta$ ( $\beta 6$ and $\beta 7$ ). Free hCG $\alpha$ (mol wt, $20 \mathrm{k}-28 \mathrm{k}$ ) can be detected only with $\alpha$-MCA. 
changes of a particular epitope. An example is the MCA specific for the epitope $\alpha 1$, which weakly stained holo-hCG but strongly reacted with the isolated $\alpha$-subunit (Fig. 1). This phenomenon has also been observed in competitive RIA (4) where this MCA displayed affinities $\left(K_{a}\right)$ for hCG and free $\mathrm{hCG} \alpha$ of $6.9 \times 10^{6}$ and $5.7 \times 10^{8}$ liter $/ \mathrm{mol}$, respectively.

IEF of holo-hCG and each of its free subunits revealed multiple bands with different pl (Fig. 2a). Approximate pl values ranged from 4 to 8 for holo-hCG, 8 to 10 for free $\mathrm{hCG} \alpha$, and 3 to 7 for free hCG $\beta$. Each pl isoform of the three standard preparations was detected by any of the respective MCA (Fig. 2a). In the presence of $8 \mathrm{~m}$ urea, holo-hCG dissociated completely into its subunits (Fig. 2b).

\section{Tumor-derived $h C G$}

From the urine of two patients (H.K., C.F.) with nonseminomatous testicular cancer, hCG variants were obtained that differed by charge (hCGav) or by size and charge (hCG $\beta$ -

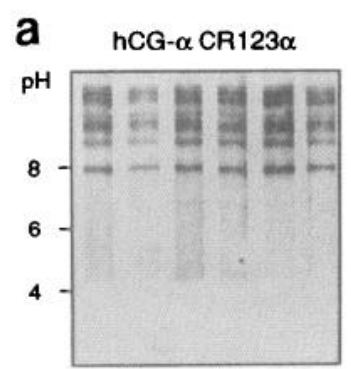

$\begin{array}{lllllll}\alpha 1 & \alpha 2 & \alpha 3 & \alpha 4 & \alpha 5 & \alpha 6\end{array}$

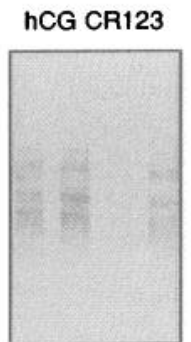

c1 $\quad$ c2 $\quad$ c3 $\quad$ c4
hCG-B CR123B

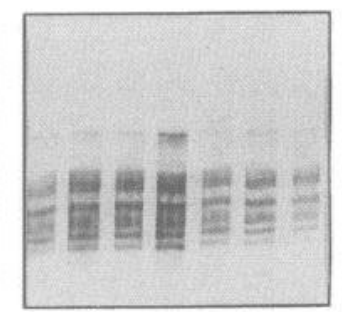

$\begin{array}{llllllll}B 1 & B 2 & B 3 & B 4 & B 5 & B 6 & B 7\end{array}$

\section{hCG CR123}

b

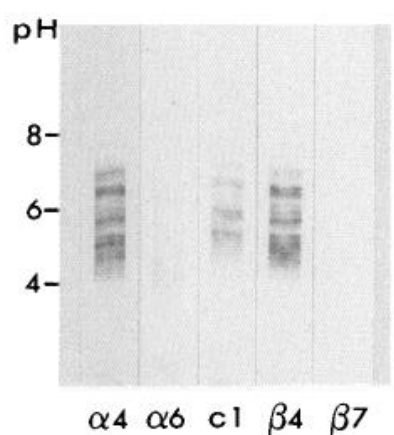

$+6 M$ urea

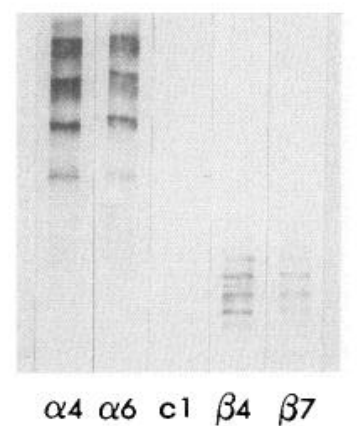

FIG. 2. Analytical IEF and Western blotting analysis of highly purified standard pregnancy hCG (NIH, CR123), free hCG $\alpha$ (NIH, CR123A), and free hCG $\alpha$ (NIH, CR123B). Immunostaining with 17 different MCA against 17 distinct epitopes of hCG. a) Antibody staining patterns with holo-hCG (c-MCA), free hCG $\alpha(\alpha-\mathrm{MCA})$, and free hCG $\beta(\beta$-MCA) are highly similar within each group. Thus, any major isoelectric variant of pregnancy hCG and of its free subunits, respectively, exposes the same immunological determinants. b) The isoforms of the intact $\alpha \beta$ heterodimer (hCG, NIH, CR 123, left panel), detected only by MCA against epitopes on the surface of holo-hCG $(\alpha 4, \mathrm{c} 1$, and $\beta 4)$, have isoelectric points of 4-8. Upon complete urea-dissociation of the holohormone (right panel), the free subunit-specific epitopes $\alpha 6$ and $\beta 7$ emerge, whereas holo-hCG-specific determinants disappear (c1). Free hCG subunits either display alkaline isoelectric points of above 8 $(\mathrm{hCG} \alpha)$ or acidic points of below $4.5(\mathrm{hCG} \beta)$. This is in accordance with higher numbers of terminal sialic acid residues on $\mathrm{hCG}_{\beta}$ as compared to $\mathrm{hCG} \alpha$. core, $\alpha$-homodimer) from hCG and free hCG-subunit standard preparations. They were analyzed by IEF, or SDS-PAGE, combined with Western blotting. Similar to the standard preparations, both molecular forms and all $\mathrm{pI}$ variants could specifically be detected with our MCA (Figs. 3 and 4).

\section{hCGav}

The preparation of hCGav (pI 3.63 to 3.93) appeared to be quite homogenous (Fig. $3 \mathrm{~b}$ ) and more acidic than the most acidic forms within standard pregnancy hCG (Fig. 3a).
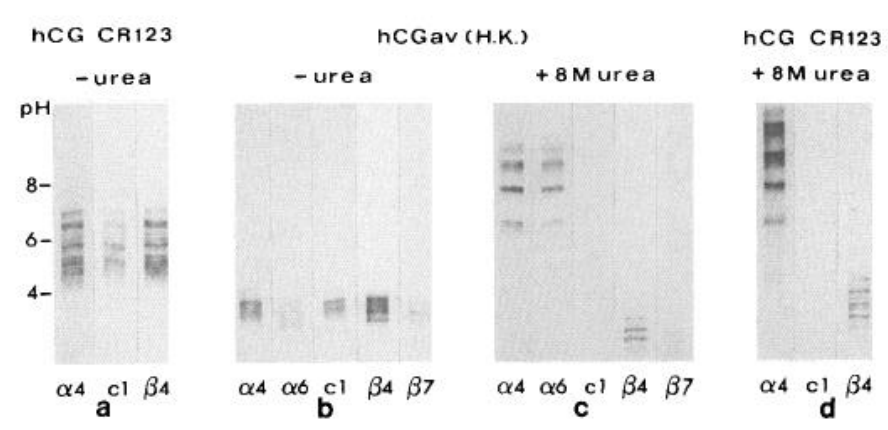

FIG. 3. IEF analysis of the highly acidic (pI 3.63-3.93) tumor-derived hCG variant (hCGav from patient H.K.) compared to pregnancy hCG (NIH, CR123). hCGav was obtained by preparative IEF and immunostained. MCA representative for each of the five main specificity groups are shown: the antibody directed against epitope $\alpha 4$ recognizes holohCG and free $\alpha, \alpha 6$ only free $\alpha, \mathrm{c} 1$ only the intact $\alpha \beta$-dimer, $\beta 4$ holo$\mathrm{hCG}$, and free hCG $\beta$ and $\beta 7$ only free hCG $\beta$. a) hCG (NIH, CR123) in the absence of urea has a pI in the range of 4-7. b) hCGav in the absence of urea: highly acidic holo-hCG (c1, $\alpha 4, \beta 4)$, as well as minor amounts of copurified acidic free subunits $(\alpha 6, \beta 7)$, could be specifically detected by our MCA. c) hCGav in the presence of urea dissociated into its free subunits with either alkaline pIs (free hCG $\alpha$ ) or acidic pIs $(\mathrm{hCG} \beta)$. d) hCG (NIH, CR123) dissociated in the presence of urea into its free subunits.

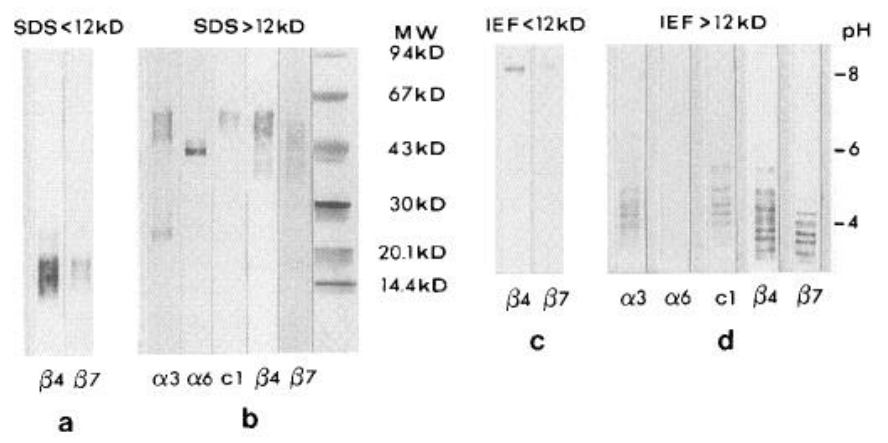

FIG. 4. SDS-PAGE (a, b) and IEF (c, d) analysis of a charge and a mol wt hCG variant from the urine of the patient C.F. Immunostaining with MCA which are representative for each of the five main antibody specificity groups is shown: the antibody directed against epitope $\alpha 3$ recognizes holo-hCG and free $\alpha, \alpha 6$ only free $\alpha, \mathrm{cl}$ only the intact $\alpha \beta$ dimer, $\beta 4$ holo-hCG and free hCG $\beta$ and $\beta 7$ only free hCG $\beta$. SDS-PAGE (a) and IEF (c) analysis of the less than $12 \mathrm{kDa}$ fraction obtained by Ultrogel AcA 44 filtration from urine. The MCA directed against epitopes $\beta 4$ and $\beta 7$ detected a highly basic molecule (hCG $\beta$-core) with an apparent mol wt of 14-20 k (SDS-PAGE) corresponding to $12 \mathrm{k}$, as determined by gel filtration. SDS-PAGE (b) and IEF (d) analysis of the urinary greater than $12 \mathrm{kDa}$ fraction containing molecules such as holo-hCG (lanes: $\alpha 3, \mathrm{c} 1, \beta 4$ ), free $\alpha$-subunit (lane: $\alpha 3$ ), free hCG $\beta$ (lanes: $\beta 4$ and $\beta 7$ ) and the $45 \mathrm{kDa} \alpha$-homodimer (lane: $\alpha 6$ ). 
HCGav consisted predominantly of holo-hCG, as demonstrated by c-MCA. Minor amounts of free $\alpha$ - and $\beta$-subunits were copurified (Fig. 3 b). Obviously a given charge can be associated with molecules of vastly different sizes, such as holo-hCG, free hCG $\alpha$ or free hCG $\beta$. After complete dissociation of hCGav by $8 \mathrm{M}$ urea (no staining with c-MCA), the pattern of $\alpha$-MCA-labeled $\mathrm{pI}$ variants appeared quite similar to that seen with the $\alpha$-subunit of dissociated pregnancy hCG, whereas $\beta$-MCA-stained $\mathrm{pI}$ isoforms were less heterogeneous and generally more acidic (Fig. $3, \mathrm{c}$ and Id). This suggests that overglycosylation or oversialylation of holohCGav had occurred on the $\beta$-rather than the $\alpha$-subunit.

\section{$h C G \beta$-core and $\alpha$-homodimer}

Analysis of the two urinary fractions of patient C.F. separated by gel chromatography on Ultrogel AcA 44 revealed two further variants of $h C G$. The less than $12 \mathrm{kDa}$ fraction contained hCG $\beta$-core (Fig. 4a), significantly smaller than standard hCG $\beta$ (see Fig. 1) and normal-sized hCG $\beta$ that was immunodetected via epitopes $\beta 4$ and $\beta 7$ in the greater than $12 \mathrm{kDa}$ fraction (Fig. 4b). HCG $\beta$-core displayed a pl in the high alkaline region (Fig. $4 \mathrm{c}$ ), where intact $\mathrm{hCG} \beta$ never occurs (Fig. 4d). Most noteworthy, in the greater than $12 \mathrm{kDa}$ fraction the MCA highly specific for the free $\alpha$-subunit $(\alpha 6)$ stained a band of much larger size than expected (Fig. $4 b$ ). However, this molecular variant, with an apparent size of 45 $\mathrm{kDa}$ is smaller than holo-hCG stained with $\mathrm{c1-MCA}$ and with $\alpha 3-\mathrm{MCA}$ (Fig. $4 \mathrm{~b}$ ). The greater than $12 \mathrm{kDa}$ fraction also contained some free hCG $\alpha$ that appeared as a band of smaller size when stained with the $\alpha 3$-MCA (Fig. 4b).

\section{Discussion}

Since pregnancy or tumor-derived variants of holo-hCG, $\mathrm{hCG} \alpha$, and $\mathrm{hCG} \beta$, often represent major fractions in body fluids, e.g. hCG $\beta$-core (18) and hCGav (17), can amount up to more than $90 \%$ and $45 \%$ of hCG immunoreactivity, respectively, the possibility that certain hCG forms might escape immunoassay detection by highly specific MCA is of major practical importance $(17,18,20,21)$. Variant forms of hCG either differ in the extent of glycosylation, the degree of terminal sialylation, and hence their net charge (12), or in their protein backbone amino acid structure (19). A main task of the present study was to clarify the involvement of oligosaccharides in epitopes expressed on hCG and their influences on antibody recognition in view of MCA overspecificity. After separation, either according to molecular size by SDS-PAGE or according to net charge by IEF, we analyzed in Western blots whether standard pregnancy and tumorderived molecular variants of hCG and of its free subunits exposed the full array of epitopes recognized by our 17 different MCA.

The present investigation provides clear evidence that all MCA against the 14 different accessible epitopes of holohCG recognized all pl variants of standard pregnancy hCG (NIH, CR123) and tumor-derived hCG. The same was true for the three MCA against the three free subunit-specific epitopes. This suggests that the overall antigenic properties of holo-hCG, hCG $\alpha$, and hCG $\beta$ are preserved on physiological and pathological isoelectric variants.

There are two asparagine $(\mathrm{N})$-linked oligosaccharides on hCG $\alpha$ (Asn 52, Asn 78), two on hCG $\beta$ (Asn 13, Asn 30) and four serine $(\mathrm{O})$-linked oligosaccharides on the $\mathrm{C}$-terminal peptide (CTP; 9, 10). Each (N)-linked carbohydrate of pregnancy hCG has been described to be potentially biantennary, and each $(\mathrm{O})$-linked unit to be monoantennary but nevertheless carrying two $\mathrm{N}$-acetyl neuraminic acids (for review see 2,11 ). Thus, hCG $\alpha$ carries maximum of 4 and hCG $\beta$ up to 12 sialic acid residues. Consequently, the overall net charge of holo hCG is primarily determined by its $\beta$-subunit.

Similar to holo-hCG, which carries up to 16 sialic acid residues, free $\beta$-subunits showed an IEF pattern covering most of the pl range ( 3 to 10 ) of our system. By contrast, free $\alpha$-subunits localized in the alkaline $\mathrm{pI}$ region corresponding to their limited number of sialic acid residues (11). Hence, there is some overlap of variants of holo-hCG and free $\beta$ subunits, in the pI range from 5 to 7 , but not of detached free hCG $\alpha$-subunits (Fig. 2a).

hCGav was identified as a tumor-derived variant with abnormally high negative surface charge predominantly localized in its $\beta$-subunit. This isoform might be based on an increased terminal sialylation and in multiantennary oligosaccharides $(22,23)$. It is not known whether hCGav represents a translational product of physiologically silent $h C G \beta$ genes. Urine from healthy pregnant women and from tumor patients contains hCG $\beta$-cure (18) a small biolugically inactive form of hCG $\beta$ lacking, among other sequences, the CTP (19). Lectin-binding studies revealed that this molecule is nearly devoid of sialic acid (24) which is consistent with its alkaline $\mathrm{pI}$ of greater than 8 . We found that purified tumor-derived hCG $\beta$-core could be labeled with all $\beta$-MCA, including those directed against the hidden subunit epitopes $\beta 6$ and $\beta 7$. [p]

Interestingly, some copurified material corresponded to an apparent size of $45 \mathrm{kDa}$ in SDS-PAGE. Since this band was stained by the $\alpha 6-\mathrm{MCA}$, which is specific for the free $\alpha$ subunit-epitope, that normally labels only a single band between 20.1 and $30 \mathrm{kDa}$, it may represent an inverted homodimer of two $\alpha$-subunits such that they can expose the normally hidden $\alpha$-subunit-epitope. This form is certainly not the intact $\alpha \beta$-heterodimer, since it could neither be stained with holo-hormone specific c-MCA nor with $\beta$-MCA. The diagnostic and clinical relevance of this molecule remains to be elucidated.

Since both normal pregnancy- and tumor-derived variants of hCG with abnormal highly negative carbohydrate-associated surface charges and variants lacking this charge were detectable with each of our MCA, it is obvious that carbohydrate moieties play no significant role in the immunological make-up of hCG. Sugar antennae, even highly branched and oversized ones, apparently do not mask epitopes, and acidic oligosaccharides do not exert electrostatic repulsive forces $v s$. antibodies, preventing their binding to the respective epitopes. Thus, epitopes of hCG seem to be predominantly shaped by the protein backbone. Glycosylation appears to have little influence on single epitopes whereas it is a crucial factor for the biological activity of $\operatorname{hCG}(2,17)$. 
These data support the diagnostic specificity, sensitivity, and accuracy of currently employed immunoassays for hCG as long as they can detect holo-hCG and free hCG $\beta$. Employing any specific MCA for accessible epitopes of hCG $\beta$ would be appropriate for the accuracy of such immunoassays. The likelihood of a false negative result for pregnancy or hCG-secreting tumors seems to be small except for cases when free $\alpha$-subunit is secreted exclusively $(5,8,25)$.

\section{Acknowledgments}

We thank Miss B. Mayregger for her skillful technical assistance. $h C G, h C G \alpha$ and hCG $\beta$ were kindly provided by the National Pituitary Agency, NIADDK (Bethesda, MD).

\section{References}

1. Hussa RO. 1981 Human chorionic gonadotropin, a clinical marker review of its biosynthesis. Ligand Rev. 3 [Suppl 2]:6-43.

2. Ryan RJ, Keutmann HT, Charlesworth MC, McCormick DJ, Milius RP, Calvo FO, Vutyavanich T. 1987 Structure-function relationship of gonadotropins. Rec Prog Horm Res. 43:383-429.

3. Mann K, Karl HJ. 1983 Molecular heterogeneity of human chorionic gonadotropin and its subunits in testicular cancer. Cancer. 52:654660.

4. Berger P, Klieber R, Panmoung W, Madersbacher S, Wolf $\mathrm{H}$, Wick G. 1990 Monoclonal antibodies against the free subunits of human chorionic gonadotrophin. J Endocrinol. 125:301-309.

5. Ridgeway EC, Klibanski A, Ladenson PW, Clemmons D, Beitins IZ, McArthur JW, Martorana MA, Zervas NT. 1981 Pure alphasecreting adenomas. N Engl J Med, 304:1254-1259.

6. Nagelberg SB, Marmorstein B, Khazaeli MB, Rosen SW. 1985 Isolated ectopic production of the free beta subunit of chorionic gonadotropin by an epidermoid carcinoma of unknown primary site. Cancer. 55:1924-1930.

7. Mann K, Siddle K. 1988 Evidence for the free beta subunit secretion in so-called human chorionic gonadotropin-positive seminoma. Cancer. 62:2378-2382.

8. Madersbacher S, Klieber R, Mann K, Marth C, Tabarelli M, Wick G, Berger P. 1992 Free $\alpha$-subunit, $\beta$-subunit of human chorionic gonadotropin (hCG), and intact hCG in sera of healthy individuals and testicular cancer patients. Clin Chem. 38:370-376.

9. Kessler MJ, Reddy MS, Shah RH, Bahl OP. 1979 Structure of $N$ glycosidic carbohydrate units of human chorionic gonadotropin. $J$ Biol Chem. 254:7901-7905

10. Kessler MJ, Mise T, Ghai RD, Bahl OP. 1979 Structure and location of the $\mathrm{O}$-glycosidic carbohydrate units of human chorionic gonadotropin. J Biol Chem. 254:7909-7914.

11. Blithe DL. 1990 Carbohydrate composition of the $\alpha$-subunit of human choriogonadotropin (hCG $\alpha$ ) and the free $\alpha$ molecules produced in pregnancy: most free $\alpha$ and some combined hCG $\alpha$ molecules are fucosylated. Endocrinology. 126:2788-2799.

12. Merz WE, Hilgenfeldt U, Brossmer R, Rehberger G. 1974 Amino acid and carbohydrate composition of human chorionic gonadotropin fractions obtained by isoelectric focusing. Hoppe-Seyler's Z Physiol Chem. 355:1046-1050.

13. Kofler R, Berger P, Wick G. 1982 Monoclonal antibodies against human chorionic gonadotropin (hCG): I. Production, specificity and intramolecular binding sites. Am J Reprod Immunol, 2:212-216.

14. Berger P, Panmoung W, Khashabi D, Mayregger B, Wick G. 1988 Antigenic features of human follicle stimulating hormone delineated by monoclonal antibodies and construction of an immunoradiometric assay. Endocrinology. 123:2351-2359.

15. Kofler R, Kalchschmid E, Berger P, Wick G. 1981 Production and characterization of monoclonal antibodies against bovine luteinizing hormone. Immunobiology. 160:196-207.

16. Schwarz S, Berger P, Wick G. 1986 The antigenic surface of human chorionic gonadotropin as mapped by murine monoclonal antibodies. Endocrinology. 118:189-197.

17. Mann K, Schneider N, Hoermann R. 1986 Thyrotropic activity of acidic isoelectric variants of human chorionic gonadotropin from trophoblastic tumors. Endocrinology. 118:1558-1566.

18. Kato Y, Braunstein G. $1988 \beta$-core fragment is a major form of immunoreactive urinary chorionic gonadotropin in human pregnancy. J Clin Endocrinol Metab. 66:1197-1201.

19. Birken S, Armstrong EG, Gawinowicz-Kolks MA, Cole LA, Agosto GM, Krichevsky A, Vaitukaitis JL, Canfield RE. 1988 Structure of the human chorionic gonadotropin $\beta$-subunit fragment from pregnancy urine. Endocrinology. 123:572-579.

20. Yazaki K, Yazaki C, Wakabayashi K, Igarashi M. 1980 Isoelectric heterogeneity of human chorionic gonadotropin: presence of choriocarcinoma specific components. Am J Obstet Gynecol. 138:189194.

21. Nwokoro N, Chen HC, Crambach A. 1981 Physical, biological, and immunological characterization of highly purified urinary human chorionic gonadotropin components separated by gel electrofocusing. Endocrinology. 108:291-299.

22. Mizuochi T, Nishimura R, Derappe $C$, Taniguchi T, Hamamoto R, Mochizuki M, Kobata A. 1983 Structure of the asparaginelinked sugar chains of human chorionic gonadotropin produced in choriocarcinoma. Appearance of triantennary sugar chains and unique biantennary sugar chains. J Biol Chem. 259:14126-14129.

23. Cole LA. 1987 The $O$-linked oligosaccharide structures are striking different on pregnancy and choriocarcinoma hCG. J Clin Endocrol Metab. 65:811-813.

24. Blithe DL, Akar AH, Wehmann RE, Nisula BC. 1988 Purification of beta-core fragment from pregnancy urine and demonstration that its carbohydrate moieties differ from those of native human chorionic beta. Endocrinology. 122:173-180.

25. Madersbacher S, Berger P, Mann K, Kuzmists R, Wick G. 1990 Diagnostic value of free subunits of serum chorionic gonadotropin in testicular cancer. Lancet. 1990 ii:630-1. 